\title{
David E. Balk: Dealing with Dying, Death, and Grief During Adolescence
}

\author{
Routledge/Taylor \& Francis Group, New York, NY, 2014, 265 pp, ISBN: 9780415534505
}

\author{
Taylor Burris ${ }^{1}$ (D)
}

Received: 4 April 2015/Accepted: 6 April 2015/Published online: 14 April 2015

(C) Springer International Publishing 2015

In his book, Dealing with Dying, Death, and Grief during Adolescence, David E. Balk offers perspective into grief counseling and child and adolescent psychotherapy when adolescents are faced with the reality of death early in life. In his early chapters, Balk addresses adolescent development and the changes that adolescents experience. Once he has established a framework for adolescence, he examines how adolescents cope with death and grief when faced with the loss of someone they care about, or are themselves faced with a life threatening illness. He discusses interventions to assist adolescents in their coping and even brings to light recent studies and statistics relevant to adolescents dealing with life crises. Death and bereavement have hugely impact adolescents' lives, and Balk's book is an educational guide for clinicians and counselors who are committed to supporting adolescents during a time when they are facing these difficult experiences for the first time in life. He gives professionals and more general readers an understanding of adolescent development, their experience with death and grief, and the tools to help adolescents cope with these events in a simple but informative manner. $\mathrm{He}$ also offers insight into the future of thanatology, the study of death, and the progressions it has made over the years.

In Chapter 1, "Adolescent Development and Serious Life Crises," Balk informs readers about the reality of adolescence and how development is affected by life crises. The world and many parents view adolescence as a time of turmoil, conflict, and distress, but in actuality this is only

Taylor Burris

tdburris@indiana.edu

1 Department of Criminal Justice, Indiana University Bloomington, 302 Sycamore Hall, Bloomington, IN 47405, USA valid for a small percentage of adolescents. Although adolescence is a perplexing time of physical, cognitive, interpersonal, and self-awareness changes, studies show that most adolescents consider their lives to be calm and satisfying. Counselors and researchers are often surprised by their resiliency in life crises, such as an adolescent becoming seriously or terminally ill, or grieving the death of someone close to them. Balk makes readers aware that, while these life crises present catalysts for growth and transformation, they also present conflicts in development. There are three phases of adolescent development: early, middle, and late. With each stage, an adolescent is expected to show growth, and the overall goal is to be able to master responsibility, intimacy, and individuality. At each stage, the adolescent has cognitive, behavioral, and affective responses. In a life crisis, any negative aspects or emotions of conflict seem to be heightened and place the adolescent in a more fragile state.

In Chapter 2, "Adolescent Development," Balk takes a further look into adolescents' development and a specific look at the physical, cognitive, and personal changes that are important to note during adolescence. During this time, they achieve reproductive maturity, develop cognitive skills that far exceed those they had in childhood, and begin to use their experiences to reflect, reason, and see other points of view. On top of all these changes, adolescents' brains are changing as well. Balk describes what has the potential to be very complex and confusing to readers, in very simple terms. The bottom line is that there are changes in the grey and white matter of the brain that allow metacognitive skills to emerge during adolescence. Throughout all of these changes is the strive for selfidentity. Balk summarizes the theories of Erikson, Marcia, and Josselon for readers to understand the extent of the process of achieving self-identity and all of the different 
views in the field of psychology. He closes the chapter with Daniel Offer who studied adolescent growth and the differences between "normal," "delinquent," "psychiatrically disturbed" adolescents. Offer found that the majority of adolescents are stable and fall into the "normal" category. Closing the chapter with Offer's theory allowed Balk to relate the information he discussed in chapter one about the myths of adolescence to this chapter. Offer's theory helps support the information he discussed in chapter one about adolescence being less of a time of turmoil and more of a time of development and growth.

Balk explains the social influences of ecological niches, such as family, peers, school, the media, and gangs in Chapter 3, "Ecological Niches for Adolescents." An ecological niche is an environment in which an organism responds to survival demands and prospects for thriving. Adolescence is all about learning, overcoming challenges, and making sense of reality; and adolescents do so within various ecological niches. Some ecological niches provide the potential for optimal development and growth while others threaten the well being of the adolescent. An adolescent's family influence depends on the family's affective involvement and responsiveness with one another, along with their ability to communicate and solve problems together. As youth progress from early to late adolescence, friendships become very important, and while peer pressure can be a negative incident, overall interaction with peers benefits adolescent maturation. Interaction with peers takes place at school, and the impact of school is different for those in early, middle, and late adolescence, which Balk points out to readers. The media, particularly the Internet and social media, occupies adolescents' lives. Balk explains the differential influences it has on those who live in urban areas and rural areas. Lastly, Balk discusses gangs in adolescence and the violations and drugs they entail. Gangs are a growing organization in the US, and he touches base on the theories of youth gangs for readers. Balk stresses the importance of adolescents' niches and even more the significance that they are interacting in positive, healthy ones.

Balk addresses how adolescents cope with life crises in Chapter 4, "Coping Responses of Adolescents." The cognitive approach is the most significantly used by scholars who study coping, and models have been developed to help others understand adolescent coping. In this chapter, Balk explains three fundamental models to understanding how adolescents cope and offers his critique on some as well. He looks at Rudolf Moos's model for understanding life crises and transitions denoting that coping with life crises involves several interacting aspects that all lead to the outcome of the crisis. Balk's main criticism is that the model ends with the outcome of the crisis. Seeing as how a crisis can end good or bad and be handled in different ways, Balk believes another part of the model should be added for the individual's future. Alexander Leighton's sociocultural model of responses to life crises focuses on certain social indications that facilitate people's coping with crises, essential strivings in life that mark one's personality and that mature over time, and the cross-section of the moment, meaning what one would consider a life crisis. John Coleman's focal model presents the idea that adolescents deal with the changes in their lives by facing them one issue at a time. Balk reveals that he has not found research that examines Coleman's focal theory applied to the lives of adolescents who are faced with unanticipated life crises, such as having a terminal illness. However, it has been applied when it comes to the sequencing of identity formation during later adolescence, meaning that adolescents deal with one formation of identity formation at a time and then, if necessary, they move on to another. It is important that Balk gives his opinion in a constructive manner, while still informing readers about other models. $\mathrm{He}$ does not demean them, leaving readers with an understanding of the different point of views and different ways to approach these matters.

Since this book is about death in adolescence, it is important to know the statistics and the primary causes of death during the adolescent years. In Chapter 5, "Principal Causes of Death during Adolescence," Balk informs readers that adolescence is among the healthiest years of a person's life. However, mortality statistics around the world show that the three primary reasons for adolescents' death all entail preventable violence. The three primary causes are accidents, homicides, and suicides. Accidents are the major cause of death, vehicular accidents and gun shot wounds in particular. The homicide rate among adolescents is alarming, especially for those in the United States. An adolescent from 15 to 24 years of age in the United States is 44 times more likely to be murdered than an adolescent in another country. Adolescent suicide rates around the world are beginning to grow so much that onethird of all nations consider adolescents to be the group most at risk for self-destructive acts. Balk brings up an important note that risks involve not only adolescents' committing suicide but also their suicide's leaving other to deal with the aftermath. Therefore it is relevant that an adolescent may also have to grieve the loss of someone they lost to suicide over the course of their development. Balk looked at the different sociological views to suicide, such as Emile Durkheim's, who categorized suicide into four types: altruistic, anomic, egoistic, and fatalistic. Other researchers believe suicide can be placed into four categories: surcease suicide, psychotic suicide, cultural suicide, and referred suicide. Wagner and Zimmerman believe suicide can be due to hopelessness, race, cyberbulling, and an overall deficient form of coping. Once the statistics and theories have been discussed, Balk concludes the chapter 
with four myths about suicide. The first myth is that people who talk about suicide do not carry out the act. The second myth is that suicidal individuals want to end their lives. The third myth is a sudden improvement in a suicidal person's emotional state means the risk of suicide has passed. The fourth and final myth Balk mentions is that people who commit suicide leave a note. These myths are highly significant to know because nonsuicidal adults and adolescents view the motives for suicide very differently than adolescents who consider killing themselves.

In the past chapter, Balk focused on the primary causes of death in adolescents. In Chapter 6, "Chronic, LifeThreatening Disease and Terminal Illness during Adolescence," he focuses on the uncommon causes of death in adolescents, such as illness. It is a rare occasion for an adolescent to get a life-threatening illness, but in the off chance the event does occur, the adolescent and his or her family do not care how rare it is. Their main focus is dealing with the crisis and finding the best professionals to treat the illness. Balk informs readers of some of the most common illnesses and the important factors to know about them. Diabetes is a common illness among adolescents, with Type 1 emerging during childhood or adolescence and Type 2 not emerging usually until middle adulthood. Type 2 has, however, been increasing among children 10-14 years old. There is no cure for diabetes, but it is rare that an adolescent or child dies from it. Due to the rapid physical, cognitive, and interpersonal changes that take place during adolescence, it is crucial that adolescents with diabetes take care of themselves. HIV/AIDS is a growing infection among the youth of Latin America, Africa, Asia, and the United States. Balk mentions statistics to imply the severity, informing readers that 15-24 year olds constituted $40 \%$ of all new HIV infections among adults worldwide in 2009. Human Papillomavirus (HPV) is the leading sexually transmitted infection among adolescents. This is concerning due to its linkage with cervical cancer. One of the most common cancers found in adolescents is Hodgkin's Lymphoma, a cancer that originates in the white blood cells, attacks lymph nodes, and spreads to others. Malignant Melanoma is another common cancer, which is a cancer of the skin. The last common cancer mentioned is Acute Lymphoblastic Leukemia, which is cancer of the white blood cells. Balk then describes the types of issues an adolescent may face when going through these illnesses, such as physical, interpersonal, cognitive, emotional, behavioral, and spiritual issues. The adolescent also faces dealing with a variety of primary and secondary losses that often stem from one another. Balk explains that in the diagnosis stage of cancer an adolescent's most common reaction is denial. In the treatment stage, adolescents often times prove to be resilient more than anything else. When it comes to the terminal stage, there can be a variety of different reactions. An adolescent may be in denial about death or come to terms with it, one may want to be alone or surrounded by family, and one may prefer peace and quiet while the other noise and bustle. It all depends on the adolescent themselves and their personality. As far as life after a treatment succeeds, it also depends on the adolescent. One might be ecstatic, while another may live in dread or with anxiety that the cancer will return. It is crucial to know what an adolescent is dealing with when they have these illnesses and how they may respond during different stages.

In Chapter 7, "Trauma," Balk addresses the physiological sense of trauma adolescents face and what a clinician or counselor should know about trauma disorders when treating an adolescent who has undergone a life crisis. Those with Post-Traumatic Stress Disorder (PTSD) repeatedly re-experience what they went through in the form of flashbacks, memories, nightmares, or frightening thoughts. PTSD can occur at any age and often begins in the 3 months following the traumatic event. The severity and duration of PTSD depends on the person and the case. Acute Stress Disorder (ASD) is a severe reaction to a traumatic event that has similarities with PTSD. Trauma that occurs in adolescence can linger into adulthood as well and cause problems. Balk then explains that there are two types of categories of trauma and the symptoms of each. Type 1 Traumas are sudden, distinct occurrings such as being kidnapped, sexually assaulted, or being shot. Type 2 Traumas are long lasting and come from repeated occurrences, such as being physically or sexually abused. It is possible for a single event to turn an ongoing trauma. If an individual uses a negative coping pattern, then secondary stressors can be added to the initial trauma, so it is crucial that adolescents are using healthy coping strategies. Balk provides readers with an example, so that they can explore the effects of a Type 1 Trauma. The example is a sniper attack that took place at a school with approximately 100 kids on the playground. Robert Pynoos, a psychologist who specializes in the effects of traumatic stress on human beings, and his team gained access to the school about a month after the attack and follow the children over the course of 14 months. They focused on the children's memory of the attack and their stress response reactions. They followed not only the kids that were on the playground at the time of the shooting but also the ones in the classrooms, in the buildings, and the ones not at school that day. Pynoos and his colleagues looked at their reactions immediately after, 6 months after, and 14 months after the shooting. Overall, they found that the children's PTSD was related to their proximity to the violence and the degree of threat to their lives. Using this example was a great way for Balk to show readers the effect trauma can have on adolescents' lives. Pharmaceutical and psychological 
treatments are recommended for dealing with trauma and PTSD. One of the most commonly used and known to be effective is cognitive behavioral therapy (CBT). CBT can come in many different forms such as exposure therapy, cognitive restructuring, or stress inoculation training. It all depends on the individual and what works for them.

Bereavement is the process of grieving and letting go of a loved one who has died. Many individuals face bereavement for the first time in life during adolescence. Over time, the knowledge of bereavement has evolved in the psychological research and theory. Balk explains many different psychologists' theories of bereavement in Chapter 8, "Bereavement," beginning with traditional models and making his way to a new way of thinking about bereavement. Freud believed grief and mourning to be normal human responses. He deemed recovery from bereavement to be done through "the grief work theory," a set of steps that aren't easy, but very possible and normal for one to work through. Erich Lindemann built on Freud's "the grief work theory" and developed the Acute Grief Syndrome. The syndrome aided in identifying common grief responses and provided clinicians with a baseline for recognizing and understanding normal grief reactions and assessing the changes over time in these reactions. John Bowlby focused on attachment theory and the effects being separated from their parents has on children. With the help of Colin Murray Parkes, they established the Phases of Mourning, in which the recovery from bereavement is a series of phrases that involve numbing to the shock of an attachment bond being shattered, yearning or searching for that person, disorganization and despair, and reorganization. J. Worden tweaked the traditional model of bereavement and added a task where the individual withdrawals emotion energy and reinvests it in another relationship. Dennis Klass presented the idea of continuing bonds, which is the idea that maintaining ties to the deceased is a common and normal response during bereavement. Margaret Stroebe created the Dual Process Model, which is a refinement of the grief work theory. This model also views bereavement as a natural process, but the coping is done by confronting the distress that is produced in bereavement while engaging in living. Although the model of bereavement has changed over time, Balk explains that the way to approach grieving remains the same. It is best to confront bereavement head on in a planned, devoted way in order for individuals to recover from their losses. After examining the history of bereavement, Balk informs readers about ways to check for symptoms of bereavement. There is the Core Bereavement Items (CBI) assessment along with The Hogan Grief Reaction Checklist (HGRC). Balk concludes the chapter with thoughts on pairing bereavement with other terms and disorders. There is a longstanding issue of associating depression and bereavement together, considering bereavement is deemed a necessary and normal act. Depression should only be considered after a timely response of grief has ensued. Balk also offers insight on what can be done with trauma or prolonged grief.

In the previous chapter, Balk lays down a framework of bereavement for readers and all the basic facts one needs to know about it. In Chapter 9, "Bereavement, Grief, and Mourning during Adolescence," he zooms onto specific cases of bereavement in adolescence. The three specific cases he examines are how adolescents react when a parent dies, a sibling dies, and a friend dies. Balk makes his opinion clear that, while these are the most common instances faced by adolescents, there are others such as losing a grandparent or a pet. Balk believes that, as time goes on and research progresses, these other topics will begin to receive attention as well. When an adolescent is faced with the loss of a parent, it can pose a threat to their development. They may experience trouble establishing a sense of belonging, trusting others and the world, and gaining a sense of mastery. Losing a parent during adolescence is deemed the most worth of studying among researchers, due to the significance of the loss and the unlikelihood that other losses would compare. Adolescents are likely to experience anxiety and depression, view themselves differently from other peers, withdraw from others, and feel a lack of control over their lives when dealing with the loss of a parent. Balk brings up the question of "Is it easier to cope with an anticipated death than it is a sudden death?" He points out the different views people have in general and the variety of research done and concludes that it is a toss up. Some research shows the grief of sudden death to be more excruciating, while other results show that an anticipated death is just as hard, if not harder to deal with. When an adolescent loses a sibling, their grief can tend to be overlooked. Not because nobody cares about them or doubts their pain, but parental grief seems to outweigh sibling grief. The areas of sensitivity when an adolescent loses a sibling are emotional responses, self-concept, grades and schoolwork, religion, and family dynamics and relationships. Adolescents are often faced with depression and anxiety, lack of interest in school, possible anger with God, and parental concern of "why isn't our remaining child more affected by this?" in the aftermath of losing a sibling. Balk concludes the chapter in discussing an adolescent's grief at the loss of a friend. His main concern is the lack of research done on adolescents' bereavement after the loss of a friend, considering a big part of developmental psychology is recognizing the importance of peers and friendship in adolescence. Losing a friend violates many of the basic assumptions for adolescents about the fairness and justice of life. A child dying is unpredictable and out of sequence, which can be very confusing to an adolescent because they have the impression that children are 
supposed to live into old age. The grief an adolescent experiences will depend on the severity of attachment they had to the friend they lost. Balk ties all of this information in by giving examples of these losses throughout the chapter so that readers can envision and integrate the information being given into real life situations.

Interventions are planned and precise efforts to produce desired outcomes. Balk devoted Chapter 10, "Interventions," to looking at interventions that can be used with life-threatened and bereaved adolescents. Before exploring numerous interventions, Balk voices his concern about an existing gap that continues to separate thanatology practitioners and researchers. He proposes a process where practitioners and researchers can collaborate together on interventions for adolescents. His process consists of a clear investment by both parties in terms of significant thinking about what they do and striving for intelligent, respectful decisions across both parties along with the engagement of both parties. Each party will listen to the other and will work openly and willingly together, discussing differences and learning from one another. Balk believes his theory could help bridge the gap between the two parties. He organizes the interventions of the chapter into a hierarchy of primary, secondary, and tertiary interventions. The goal of primary intervention is to strengthen individuals and/or avoid harm. The goal of secondary intervention is to enable individuals to adapt positively to life situations that may worsen without such involvement. The goal of tertiary intervention is to assist individuals who are dealing with difficult issues that have already played a role in their lives. Thanatology's primary interventions consist of formal education, in the hopes that it will aid in averting the development of complicated grief. These formal education tactics consist of college programs that promote spiritual development, information campaigns, public policy efforts, and growth and transformation based off the exploration of life values. One of the main secondary interventions is grief counseling. If one is dealing with a bereaved family, family-focused grief therapy may be of value. There are also two secondary interventions for bereaved college students. The first is the National Students of AMF, a nonprofit organization that helps college students who are coping with loss. The other one is an intervention that tests the effectiveness of providing bereaved students with social and educational support about coping. There are tertiary interventions aimed at trauma and grief, complicated grief disorder, adolescents with cancer, and palliative care for the terminally ill. Cognitive-behavioral therapy is an effective way to prevent the onset of PTSD, lessen the effect of ASD, and treat complicated grief. When an adolescent has cancer, the treatment is aimed for remission along with survival that is free of pain and the reemergence of cancer. Palliative care for the terminally ill is the management of pain. When an adolescent is dying, hospice may be brought into ensure that that individual passes away without any pain. All of the interventions Balk provides are practical steps that will assist in the lives of grieving adolescents or those that have life-threatening illnesses.

In Chapter 11, "Beyond Websites," Balk faces the reality of adolescents and the media. Adolescents who are alive today are immersed into a technological world. The Internet is not something that they use as simply a resource, but rather as an essential part of their daily lives. Technology has changed the game of adolescent communication, education, and socialization. Although it is such a big part of adolescents' lives, technology is rarely looked at or studied in a positive sense. There has been little research done over the positive impact it may have on adolescents' lives. Their technological use, specifically the Internet, is often frowned upon. However, in recent years, fields of study like cyberpsychology have emerged, and thanatologists have been working to understand and use technology in a healthy way and as a supportive system for lifethreatened and bereaved adolescents. Due to this drastic change of adolescents becoming technologically based, it is important to recognize this, especially when it comes to educating and treating these adolescents. The majority of adolescents now have access to media 24/7, due to either the Internet, cell phones, or both. Balk suggests ways to deal with this new reality and ways to integrate it into adolescents' treatment in a positive way. Adolescents are not just surfing the web looking at websites, many are involved in social networking sites. Social media is important to adolescents, so when treating someone dealing with grief or loss, a social networking site can be used as a supportive resource. With that said, before recommending a website to an adolescent, the clinician/counselor needs to do a full inspection of the site to ensure that it is going to meet the needs of the adolescent. It is vital that one checks the content and also looks at the language used to make sure it is not too childish for the adolescent. Once again, Balk brings up the Dual Process Model of coping. The model operated back and forth of dealing with loss orientation and restoration orientation. Loss orientation is confronting coping, while restoration orientation is avoiding coping. Social networking and the Internet can be used to facilitate the oscillation between loss orientation and restoration orientation. Possibly more controversial than social networking is adolescents' use of online gaming. When online gaming, adolescents get the chance to participate in a virtual world which is in a sense an escape from reality. Online gaming is often used as a means for adolescents with life-threatening illnesses. When they are playing games online and participating in virtual worlds, they get to create avatars. Their avatars allow them to be strong and 
healthy, while giving them a release from their own reality for the time being. Although these are all ways to incorporate the Internet and social media into adolescents' treatment, it is important to remember that Internet safety is still a very large concern for adolescents. Giving away too much information can be threatening to the individual's life. There is also the concern of adolescents becoming dependent on technology. While there are these negative factors, it is important to focus on the positive impacts technology can have in a bereaved adolescent's life, such as the ability to connect with others, raise social awareness, and promote empathy.

In his concluding and twelfth chapter, "Some Final Thoughts," Balk discusses the hopes he has for the future of thanatology and those involved in the field who are contributing to the understanding of grieving and dying during adolescence. Balk offers ideas he has for a total of three projects. Two of the projects are research studies and one is an intervention that combines creative arts expression and bereavement support. Balk's intervention idea is to use creative arts to teach mental health counselors, student life professionals, and school psychologists essentials about human bereavement and grief across cultures. He hopes that it will teach them how to use creative arts effectively when working with individuals who are bereaved and to apply their knowledge about creative arts not only to help the individual, but the individual's family and their surrounding community. Lastly, Balk hopes that his intervention will drive professionals and students to continuously study and further their knowledge about all different cultures on the topic of bereavement, grief, coping, and resiliency in adolescents. Balk's two research studies are about the degree of attachment the individual had to the person they lost and measuring the transformation and growth of adolescents in response to a loss. It is important to point out that, in this chapter, Balk references other studies he has done relating to these topics. He points out the strong and weak points of his studies for future researchers to learn from. Balk's analyses reveal how his interest in the future of thanatology and adolescents' lives is sincere, and how he hopes for nothing but progress in the field.

Overall, Balk expertly provides information about death, dying, and grief in adolescence for not only professionals but also the general public. This book is one that a clinician or counselor could read to gain valuable information about treating a client, or a parent who is searching for basic information and understanding about their own grieving adolescent, or just an every day reader who is interested in the topic. Balk writes it in a way that is simple but effective for anyone who chooses to read it. His opening chapters provide a framework of adolescence, touching base on what it means to be an adolescent and the changes they go through. He devotes chapters to defining the life crises adolescents' experience, along with the trauma, bereavement, and coping that follows life crises. Although some of the beginning chapters have materials that are repetitive, Balk is trying to provide a clear and basic understanding for the readers on adolescents and what they go through when they are terminally ill or lose a loved one. Once the readers have an understanding of what they need to know, Balk delves into more complex materials in chapters mentioning models that exist and are being developed for coping strategies, trauma, bereavement, treatments, and interventions for adolescents. Balk does not just offer his expertise, he offers the guidance of many other professionals and the work they have done in the study of adolescents on this topic. He looks at statistics and recent research to provide the most up to date information he can. He takes into consideration that individuals will grieve and deal with life crises in different ways and also that all professionals are unique in their ways of providing treatment as well. Due to this, he provides a variety of different interventions that can work for a variety of different individuals. Balk focuses on the positive and negative aspects of interventions and what can be done in future research to advance the field of psychology when studying dying, death, and grief in adolescence. 